\title{
Anemia hemolítica del recién nacido secundaria a incompatibilidad del subgrupo Kell
}

\author{
Hemolytic anemia in the newborn secondary \\ to subgroup Kell incompatibility \\ Dennise Crespo Smith, ${ }^{*}$ José Manuel Septién Guevara, ${ }^{\ddagger}$ Olivia Eloísa Ortiz Ramírez§
}

\section{RESUMEN}

La anemia en el recién nacido se puede presentar por diferentes causas, una de ellas es la enfermedad hemolítica del recién nacido, la cual puede ser secundaria por incompatibilidad al $\mathrm{Rh}$ o $\mathrm{ABO}$; sin embargo, existen subgrupos en quienes también puede originarse una incompatibilidad y secundariamente una anemia hemolítica, ictericia e hiperbilirrubinemia. Esta última se presenta con menor frecuencia, el cuadro clínico es más severo y requiere de la atención del recién nacido en una unidad de cuidados intensivos neonatales. Presentación del caso: Este trabajo describe el caso de un recién nacido que desde el nacimiento muestra palidez, escaso esfuerzo respiratorio y desaturaciones. Se ingresa a la unidad de cuidados intensivos neonatales por continuar con palidez y desaturaciones, requiriendo de apoyo con oxígeno. Desde su ingreso se encuentra con anemia, hiperbilirrubinemia y hepatomegalia, por lo que se decide realizar exanguinotransfusión y se solicita valoración a hematología, encontrando incompatibilidad al subgrupo Kell. La mayoría de los casos por incompatibilidad al subgrupo Kell desarrollan una anemia hemolítica severa y una evolución con mal pronóstico. Conclusión: En este caso podemos ver la buena evolución del recién nacido gracias a un manejo oportuno y la importancia de conocer otras causas de anemia hemolítica en el recién nacido.

Palabras clave: Anemia hemolítica, incompatibilidad al subgrupo Kell, ictericia del recién nacido.

Nivel de evidencia: IV

\begin{abstract}
The anemia of the newborn can be from different causes, one of them is the hemolytic anemia of the newborn which can be secondary to Rh o ABO incompatibility, however there exist other subgroups that can cause incompatibility and secondary hemolityc anemia, jaundice and hyperbilirubinemia. This one presents with less frequency and may result in progressive anemia and it requires attention of the newborn in a neonatal intensive care unit. Case report: This work describes the case of a pale newborn with poor respiratory effort and low oxygen rates, with anemia, hyperbilirubinemia and hepatomegaly who requires exchange transfusion and evaluation of the hematologist who found incompatibility to subgroup Kell. Most of the cases due to subgroup Kell incompatibility present a severe hemolytic anemia with a fatal evolution and not a good prognosis. Conclusion: In this report we can see that the newborn had a satisfactory evolution due to prompt treatment and the importance of knowing different causes of hemolytic anemia of the newborn.
\end{abstract}

Keywords: Hemolytic anemia, subgroup Kell incompatibility, jaundice of the new born.

Level of evidence: $I V$

\section{* Pediatría y Neonatología. \\ ‡ Ginecología y Obstetricia. \\ § Pediatría. Grupo Pediátrico de Santa Fe, S.C.}

Centro Médico ABC, Santa Fe.

Recibido para publicación: 05/12/2019. Aceptado: 04/04/2020.

Correspondencia: Dennise Crespo Smith

Carlos Graef Fernández Núm. 154, consultorio 1A,

Col. Tlaxala, 05300, Cuajimalpa, CDMX.

E-mail: decres@gmail.com

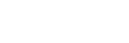

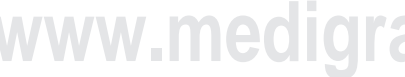




\section{Clasificación de la anemia en el recién nacido}

- Falla en la producción de glóbulos rojos (anemia del prematuro, anemia tardía secundaria a hemólisis por incompatibilidad al Rh, deficiencia neonatal de hierro y anemia de Diamond-Blackfan).

- Hemorragia (prenatal, perinatal y postnatal).

- Hemólisis (inmunológicas: Rh, ABO, Kell, Kidd, Duffy, MNS), autoinmunidad materna. Enfermedades adquiridas no inmunológicas: infección por citomegalovirus, toxoplasmosis, sífilis, sepsis, coagulación intravascular diseminada. Mutaciones del citoesqueleto del eritrocito: esferocitosis hereditaria, eliptocitosis hereditaria, entre otras. Anomalías en las enzimas del eritrocito: deficiencia de glucosa 6-fosfato deshidrogenasa, deficiencia de piruvato kinasa, talasemia alfa y gamma y hemoglobinas inestables. ${ }^{1}$

- La hemólisis secundaria por incompatibilidad a $\mathrm{ABO}$ se presenta en $12 \%$ de los embarazos, el cuadro clínico es moderado y se manifiesta con hiperbilirrubinemia, pero no con anemia ni con hidrops. Sin embargo, la enfermedad hemolítica por ABO ha sido descrita como la principal causa de hiperbilirrubinemia extrema y Kernicterus. ${ }^{2}$ La mayoría de los casos son tratados con fototerapia y rara vez exanguinotransfusión. Dentro de las causas de ictericia patológica en el recién nacido están las de origen hemolítico y dentro de ellas las inmunitarias son la mayoría. Éstas se manifiestan en las primeras horas de vida y son a consecuencia de una incompatibilidad entre el grupo materno y el del recién nacido, siendo los grupos $\mathrm{Rh}$ y $\mathrm{ABO}$ los más frecuentemente involucrados. La incompatibilidad por grupo Kell es una de las menos frecuentes (menos de 5\% de las anemias hemolíticas en conjunto con la incompatibilidad por grupo C y E). ${ }^{3}$

- La enfermedad hemolítica por incompatibilidad al subgrupo Kell es una causa importante de hemólisis del recién nacido, ${ }^{4,5}$ es la tercera causa de hemólisis en el recién nacido después de la incompatibilidad al Rh y ABO. El gen Kell se localiza en el cromosoma 7 (7q34). ${ }^{6,7}$ El sistema Kell se llamó así en honor a la primera mujer en quien se detectó este anticuerpo. Cuando los anticuerpos maternos son expresados a este antígeno, ataca a progenitores eritroides y crea lo que conocemos como anemia hipoproliferativa. Se llama hipoproliferativa porque el recuento de reticulocitos es bajo o normal y la hiperbilirrubinemia no es tan severa. Un recién nacido con enfermedad hemolítica anti-K presenta anemia severa sin reticulocitosis e hiperbilirru- binemia. El cuadro clínico se puede confundir en un inicio con pérdida sanguínea fetal como una transfusión fetoplacentaria. ${ }^{6} \mathrm{El}$ anticuerpo Kell 1 se presenta en 1/1,000 mujeres embarazadas. La mayoría de ellas desarrollan este anticuerpo posterior a una transfusión. El anti-K puede generar reacciones hemolíticas graves y enfermedad hemolítica del recién nacido; junto con anti-D, C y E son las principales causas de enfermedad hemolítica del recién nacido clínicamente significativas. ${ }^{8-10}$

\section{PRESENTACIÓN DEL CASO}

Mujer de 30 años, con cinco gestas, un parto, dos cesáreas, un aborto, grupo y $\mathrm{Rh} \mathrm{O}$ positivo. Tiene tres hijos vivos y sanos, quienes no tuvieron complicaciones al nacimiento.

Durante el embarazo actual se diagnosticó diabetes gestacional mediante curva de tolerancia oral a la glucosa de $120 \mathrm{~min}$ ( $75 \mathrm{~g}$ ) a la semana 26 con valores de $93 \mathrm{mg} / \mathrm{dL}$ en ayunas, $129 \mathrm{mg} / \mathrm{dL}$ a la hora y $140 \mathrm{mg} / \mathrm{dL}$ a las dos horas. Tratamiento con dieta de 1,700 calorías, con adecuado control, última glucosa pre- y postprandial a la semana 32 de $90 / 115 \mathrm{mg} /$ $\mathrm{dL}$, respectivamente. Infección de vías urinarias con urocultivo positivo para estreptococo del grupo B tratado con ampicilina y urocultivo de control negativo.

Ultrasonido obstétrico a la semana 13 y 21 con placenta que cubre completamente el orificio cervical interno, por lo que de nuevo se realizó ultrasonido con vejiga llena y por vía endovaginal a la semana 28 , encontrando placenta previa total y a la exploración con Doppler color se observan datos de acretismo placentario sin descartar infiltración vesical.

Por este motivo se prescribe esquema de madurez pulmonar fetal con betametasona $12 \mathrm{mg}$ intramuscular cada 24 horas por dos dosis a la semana 28 y se solicita resonancia magnética.

La resonancia magnética se efectuó a la semana 32, la cual revela placenta bilobulada con mayor componente posterior, placenta previa, acretismo placentario focal a nivel de segmento anterior y tercio medio del componente posterior, sin datos de placenta percreta.

Presenta sangrado transvaginal abundante a la semana 34.3 (primer evento de sangrado en embarazo actual), aumento del tono uterino y dolor, producto en situación longitudinal, presentación cefálica con frecuencia cardiaca fetal de 140 por minuto. Se realiza especuloscopia en la que se observa canal vaginal con sangre y coágulos, así como sangrado activo a través del orificio cervical externo, por lo que se decide interrupción del embarazo vía abdominal. 
Se procede a cesárea corporal e histerectomía total abdominal con conservación de anexos. Tuvo un sangrado transoperatorio de $1,000 \mathrm{~mL}$ con hemoglobina prequirúrgica de $10.1 \mathrm{~g} / \mathrm{dL}$ y hemoglobina postquirúrgica de $8.8 \mathrm{~g} / \mathrm{dL}$, no hubo descompensación hemodinámica, por lo que no requirió transfusión sanguínea ni el ingreso a cuidados intensivos. Se administró cefalotina previo al inicio de la cirugía. No hubo complicaciones durante el procedimiento y la evolución postoperatoria fue favorable.

El resultado de patología fue: útero de 1,550 gramos, miometrio hipertrófico con ectasia de los vasos sanguíneos (atonía uterina), placenta previa total con acretismo placentario del tipo placenta increta con afectación principal del istmo y cervicitis crónica con metaplasia escamosa inmadura.

Nace producto del sexo femenino a las 34.3 semanas de gestación a través de cesárea por diagnóstico de placenta previa total y acretismo anterior y posterior. Llora y respira al nacer, se coloca en cuna radiante y se inician maniobras básicas de reanimación, posteriormente presenta palidez, esfuerzo respiratorio deficiente y bradicardia menor de 70 latidos por minuto, por lo que se decide iniciar ventilación con presión positiva requiriendo de dos ciclos con buena recuperación de la frecuencia cardiaca; sin embargo, continúa con desaturación, por lo que se coloca oxígeno indirecto y se aspiran abundantes secreciones, se pinza y corta cordón umbilical y se decide su ingreso a la unidad de cuidados intensivos neonatales. Se califica con Apgar 7/8 y Silverman Andersen de 3 a expensas de tiraje intercostal y aleteo nasal.

A su ingreso se coloca casco cefálico, catéteres umbilicales; glucómetro en $73 \mathrm{mg} / \mathrm{dL}$ y gasometría arterial, la cual se encuentra en equilibrio ácido base y reporta hemoglobina de $7.5 \mathrm{mg} / \mathrm{dL}$. Se decide pasar una carga de solución fisiológica a $20 \mathrm{~mL} / \mathrm{kg}$.

A la exploración física con peso $2,320 \mathrm{~g}$, talla 44 $\mathrm{cm}$, perímetro cefálico $33 \mathrm{~cm}$, perímetro torácico 30 $\mathrm{cm}$, perímetro abdominal $30 \mathrm{~cm}$. Frecuencia cardiaca 175 por minuto, frecuencia respiratoria 83 por minuto, TA $96 / 56 \mathrm{mmHg}$ y temperatura $36{ }^{\circ} \mathrm{C}$, palidez de tegumentos, quejido respiratorio, abdomen blando, depresible, no doloroso a la palpación, se palpa hígado a $3 \mathrm{~cm}$ por debajo de borde costal derecho.

Se realizaron los siguientes laboratorios: biometría hemática con hemoglobina $8.1 \mathrm{~g} / \mathrm{dL}$, hematocrito $23.3 \%$, plaquetas $215 \mathrm{mil} / \mathrm{mm}^{3}$, leucocitos $8.4 /$ $\mathrm{mm}^{3}$, linfocitos $45 \%$, segmentados $30 \%$, bandas $9 \%$, eosinófilos $7 \%$, monocitos $5 \%$. En las pruebas de función hepática se reportan bilirrubinas totales de $7.7 \mathrm{mg} / \mathrm{dL}$, bilirrubina directa $0.60 \mathrm{mg} / \mathrm{dL}$, bilirrubi- na indirecta $7.1 \mathrm{mg} / \mathrm{dL}$, creatina-fosfoquinasa (CPK $213 \mathrm{mg} / \mathrm{dL}$ ), procalcitonina $0.92 \mathrm{ng} / \mathrm{mL}$. Grupo y Rh O positivo, Coombs positivo.

Con los resultados anteriores se inicia fototerapia con protección ocular y se solicita interconsulta con hematología por la presencia de hemólisis, la cual se manifestó con anemia, hiperbilirrubinemia y hepatomegalia.

Se realiza estudio inmunohematológico, el cual reveló lo siguiente: grupo $(\mathrm{ABO})$ : $\mathrm{O}$, factor $\mathrm{Rh}$ : negativo, du tubo (determinación del antígeno D Débil$\mathrm{Du}$ ): negativo, fenotipos: K positivo, Coombs directo: positivo y anticuerpos irregulares: negativo.

Se decide efectuar el mismo día del nacimiento exanguinotransfusión con recambio a doble volumen. Al terminar el procedimiento se reporta una hemoglobina de $13.6 \mathrm{mg} / \mathrm{dL}$ y horas después de 12.4 $\mathrm{mg} / \mathrm{dL}$ así como bilirrubina total de $5.6 \mathrm{mg} / \mathrm{dL}$.

Se realiza estudio inmunohematológico a los padres, el cual reveló lo siguiente:

- Padre del recién nacido: grupo (ABO): O, factor $\mathrm{Rh}$ : positivo, fenotipos: $\mathrm{K}$ positivo y anticuerpos irregulares: negativo.

- Madre del recién nacido: grupo (ABO): $\mathrm{O}$, factor $\mathrm{Rh}$ : positivo, Coombs: positivo, fenotipos: $\mathrm{K}$ positivo $\mathrm{y}$ anticuerpos irregulares: positivo (anti-K 1/256).

\section{DISCUSIÓN}

Los principales antígenos involucrados en la enfermedad hemolítica del recién nacido son los que pertenecen al sistema $\mathrm{Rh}$, el cual incluye los antígenos D, c, C, E y e. A pesar del desarrollo de métodos para prevenir la isoinmunización materna a los antígenos $\mathrm{Rh}$ (D) es una causa importante de anemia e hiperbilirrubinemia en los recién nacidos. Esta enfermedad se asocia principalmente con antígeno $\mathrm{D}$ del grupo $\mathrm{Rh}$ en $50 \%$ y rara vez es causada por antígeno $\mathrm{C}$ o $\mathrm{E}$, o por otros como Cw, Cx, Du, K (Kell), M, Duffy, S, P, MNS, Xg, Lutteran, Diego y Kidd. ${ }^{11}$

La capacidad de las mujeres $\mathrm{Rh}$ negativas para formar anticuerpos es variable, por lo tanto la incidencia general para la isoinmunización es baja, con anticuerpos contra el antígeno D detectados en menos de $10 \%$ aun después de cinco embarazos o más; sólo 5\% tendrá hijos con enfermedad hemolítica. ${ }^{11}$

Dentro de los factores que influyen en el desarrollo de anticuerpos anti-Rh D está la placenta previa como lo fue en este caso, además de otros factores como el embarazo ectópico, desprendimiento de placenta, etcétera. 
En este caso el recién nacido presentó anemia e hiperbilirrubinemia desde el nacimiento, con un cuadro clínico franco de hemólisis, motivo por el cual se decidió efectuar el procedimiento de exanguinotransfusión dentro de las primeras 12 horas de vida con una respuesta clínica favorable y sin complicaciones. Se realizaron estudios inmunohematológicos a los padres, quienes reportan incompatibilidad al antígeno Kell. Cuando existe incompatibilidad al subgrupo Kell, la principal manifestación es una anemia severa, y menos frecuente la hiperbilirrubinemia en comparación con incompatibilidad al $\mathrm{Rh}$ o a otros tipos de incompatibilidad anti-D. ${ }^{12}$

Si existe una isoinmunización importante durante el embarazo y no se da un tratamiento adecuado se presenta anemia fetal progresiva, hidrops fetal, asfixia y muerte perinatal. Después del nacimiento la hiperbilirrubinemia puede progresar a kernicterus y causar daño neurológico, problemas de audición y en el desarrollo psicomotor.

La enfermedad hemolítica severa del recién nacido secundaria a incompatibilidad al subgrupo Kell se puede manifestar en etapas tempranas del embarazo y provocar un desenlace fatal en el recién nacido, por eso la importancia de realizar estudios serológicos y moleculares a los padres pensando en un futuro embarazo.

\section{DISCUSIÓN Y RESULTADOS}

Este trabajo describe el caso de una hemólisis de recién nacido secundaria a una destrucción inmune de eritrocitos por el aloanticuerpo contra la glucoproteína Kell.

Estudios serológicos maternos y paternos demuestran la presencia del aloanticuerpo. En la mayoría de los casos cuando existe la presencia de anticuerpos del subgrupo Kell, el cuadro clínico puede ser severo y causar supresión de la eritropoyesis. Por lo general se manifiesta con anemia y no con hiperbilirrubinemia, en este caso se presentaron ambas; en comparación con casos de anemia hemolítica causados por anti-D u otros tupos o por el Rh. ${ }^{12}$ Por la considerable hiperbilirrubinemia en las primeras horas de vida, la anemia y la prematurez se decidió realizar la exanguinotransfusión, que no es lo más común en cuanto al manejo de estos casos; ya que en los casos reportados el tratamiento responde solamente a la vigilancia del recién nacido y al uso de fototerapia. La evolución clínica del recién nacido en la unidad de cuidados intensivos neonatales fue muy buena, se realizó ultrasonido transfontanelar, el cual se reportó normal, ecocardiograma de control normal, cardiovascular sin alteraciones, se inició la vía oral por sonda orogástrica, la cual se fue incrementando con buena tolerancia hasta succionar adecuadamente a libre demanda. Bilirrubinas séricas dentro de parámetros normales, buena hidratación y buena coloración de tegumentos, completó esquema de antibióticos y los cultivos fueron negativos. Gracias a su buena evolución y mejoría clínica se decide su egreso a los 12 días de vida extrauterina.

\section{CONCLUSIÓN}

En conclusión, es muy importante realizar pruebas tempranas en el embarazo para encontrar antecedente de isoinmunización y así detectar embarazos con alto riesgo de hemólisis en el feto o en el recién nacido; así como conocer el comportamiento clínico de los aloanticuerpos contra los diferentes subgrupos, en este caso contra el subgrupo Kell e iniciar lo más pronto posible el tratamiento.

\section{BIBLIOGRAFÍA}

1. Gleason C, Juul S. Avery's diseases of the newborn. 10th ed. Philadelphia, PA: Elsevier; 2018.

2. Garratty G. Drug-induced immune hemolytic anemia. Hematology Am Soc Hematol Educ Program. 2009; 73-79.

3. Zuñiga M, Varela J, Valero M, Novik V, Peña C. Ictericia e Incompatibilidad por grupo Kell: caso clínico. CIMEL. 2009; 14 (2): 116-119.

4. Denomme GA. Kell and Kx blood group systems. Inmunohmatology. 2015; 31 (1): 14-19.

5. Karagol BS, Zenciroglu A, Okumus N, Karadag N, Dursun A, Hakan N. Hemolytic disease of the newborn caused by irregular blood subgroup (Kell, C, c, E, and e) incompatibilities: report of 106 cases at a tertiary-care centre. Am J Perinatol. 2012; 29 (6): 449-454.

6. Vásquez Rojas M, Castillo Espinosa D, Pavez Espinoza Y, Maldonado Rojas M, Mena Leiva A. Frecuencia de antígenos del sistema sanguíneo Rh y del sistema Kell en donantes de sangre. Rev Cubana Hematol Inmunol Hemoter. 2015; 31 (2): 160-171.

7. Scharberg EA, Wieckhusen C, Luz B, et al. Fatal hemolytic disease of the newborn caused by an antibody to KEAL, a new low-prevalence Kell blood group antigen. Transfusion. 2017; 57 (1): 217-218.

8. Aristizábal JM, Torres JD. Transfusiones en pacientes con pruebas de compatibilidad positivas y en aquellos con anemia hemolítica autoinmune. IATREIA. 2007; 2 (4): 379-387.

9. Romero D, Hernández J. Enfermedad Hemolítica del recién nacido. Rev Med Inst Mex Seguro Soc. 2005; 4 3(S1): 33-36.

10. Slootweg YM, Lindenburg IT, Koelewijn JM, Van Kamp IL, Oepkes D, De Haas M. Predicting anti-Kell-mediated hemolytic disease of the fetus and newborn: diagnostic accuracy of laboratory management. Am J Obstet Gynecol. 2018; 219 (4): 393.e1-393.e8.

11. Diagnóstico y tratamiento de la enfermedad hemolítica por isoinmunización a $\mathrm{Rh}$ en el recién nacido. Guía de Evidencias y Recomendaciones: Guía de Práctica Clínica. México, CENETEC; 2018.

12. Mattaloni SM, Amoni C, Céspedes R, Nonaka C, Trucco Boggione C, Luján Brajovich ME et al. Clinical significance of an alloantibody against the Kell blood group glycoprotein. Transfus Med Hemother. 2017; 44 (1): 53-57. 